\title{
Development and Regulation of Dendritic Stratification in Retinal Ganglion Cells by Glutamate-Mediated Afferent Activity
}

\author{
Stefan R. Bodnarenko, ${ }^{a}$ Gayathri Jeyarasasingam, and Leo M. Chalupa \\ Section of Neurobiology, Physiology and Behavior, the Center for Neuroscience, and the Department of \\ Psychology, University of California, Davis, California 95616-0657
}

In the mature retina, the dendrites of retinal ganglion cells (RGCs) are segregated into either ON or OFF sublaminae of the inner plexiform layer (IPL), but early in development the dendritic processes of these cells are multistratified, ramifying throughout the IPL. We examined the time course of dendritic stratification in developing beta cells, the largest class of ganglion cells in the cat retina, by retrograde labeling of fixed tissue with Dil. Dendritic stratification begins in the central and peripheral retina by embryonic day 50 , about 2 weeks before birth and is not fully completed until 5 months postnatally. A clear central-to-peripheral gradient in the incidence of stratified beta cells first becomes evident shortly after birth. This stratification process was effectively halted by short-term intraocular injections (4-11 d) of the glutamate analog 2-amino-4-phosphonobutyrate (APB), which hyperpolarizes rod bipolar cells and ON cone bipolar cells, thereby preventing the release of glutamate by these interneurons. APB treatment did not alter the somal sizes or the tangential extent of the dendrites of developing beta cells, nor did it cause abnormal loss of these neurons. The organization of the inner nuclear layer, containing the APB-sensitive bipolar cells, was also not compromised by such injections. When APB treatment was discontinued there was a rapid resumption of dendritic stratification resulting in a normal incidence of stratified RGCs. Thus, short-term APB treatment causes a delay rather than a permanent arrest of the stratification process. These results suggest that glutamate-mediated afferent activity regulates the restriction of developing RGC dendrites into ON and OFF sublaminae of the IPL and that such regulation takes place during a protracted developmental period.

[Key words: retinal ganglion cells, dendritic development, glutamate-mediated activity, 2-amino-4-phosphonobutyrate (APB), ON-OFF pathways, morphological plasticity]

All information about the visual world is conveyed to the brain by retinal ganglion cells (RGCs). Because a great deal is known about the structure and function of mature RGCs, and because

Received May 3, 1995; revised June 21, 1995; accepted July 5, 1995.

This work was supported by EY03991 from the NEI and NSF Grant IBN 12593

Correspondence should be addressed to Leo M. Chalupa, Psychology Department, University of California, Davis, CA 95616.

apresent address: Department of Psychology, Clark Science Center, Smith College, Northampton, MA 01063.

Copyright $(\mathcal{C} 1995$ Society for Neuroscience $0270-6474 / 95 / 157037-09 \$ 05.00 / 0$ the retina is readily accessible to experimental manipulations, these neurons have proved to be exceptionally well-suited for developmental investigations. For the most part, recent efforts have focused on the refinements of RGC axonal projections, and a number of studies have now demonstrated the importance of activity-mediated events in the establishment of the precise connectional patterns evident in mature RGCs (Dubin et al., 1986; Kalil et al., 1986; Shatz and Stryker, 1988; Sretavan et al., 1988). As yet, however, little is known about the factors regulating the growth and restructuring of RGC dendritic processes. This issue is of fundamental importance because the dendritic morphology of mature RGCs has been related to distinct visual response properties (reviewed in Wässle and Boycott, 1991).

One feature of dendritic morphology that has been correlated with a functional role is the stratification of RGC dendrites within different sublaminae of the inner plexiform layer (IPL). RGCs with dendrites that stratify in the inner portion of the IPL signal information about increments of light, while those with dendrites stratifying in the outer portion of the IPL signal light decrements (Famiglietti and Kolb, 1976; Nelson et al., 1978). Whereas in the Inlature cal retina the dendrites of ON and OFF alpha and beta RGCs are unistratified, early in development the dendritic processes of these neurons have been noted to ramify throughout the IPL (Maslim and Stone, 1988; Dann et al., 1988; Ramoa et al., 1988). Although, the precise time course of the stratification process has yet to be determined, it has been reported in the developing cat retina that this occurs around the time that bipolar cells form synapses in the IPL (Maslim and Stone, 1986, 1988). The temporal coincidence of these two developmental events suggested to us that afferent activity may be involved in the remodeling of initially multistratified RGC dendrites. Support for this idea was provided recently by the finding (Bodnarenko and Chalupa, 1993) that the remodeling of RGC dendrites, from a multistratified to a unistratified state can be disrupted in newborn cats by intraocular injections of the glutamate analog 2-amino-4-phosphonobutyrate (APB). In the mature retina APB hyperpolarizes rod bipolar and ON cone bipolar cells (Slaughter and Miller, 1981; Bolz et al., 1984; Müller et al., 1988), which blocks the release of glutamate by these interneurons (Wässle et al., 1991). On this basis, we interpreted the effects of APB treatment as suggesting that glutamate-mediated afferent activity regulates the formation of segregated ON and OFF pathways.

In the present study we have extended our investigation of the development of RGC dendritic stratification by addressing three related issues. First, we describe the time course of this regressive event in beta cells, the major ganglion cell class in the cat retina. We have found that the stratification of beta cell 
dendrites begins about 2 weeks before birth and that such remodeling is not fully completed until several months after birth. Second, we show that APB treatment, which perturbs the stratification of dendrites in beta RGCs, does not affect the survival or the size of beta cell somas and dendrites. Furthermore, APB treatment does not appear to compromise the organization of the inner nuclear layer (INL), containing the APB-sensitive bipolar cells. Third, we demonstrate that APB treated RGCs retain a remarkable degree of morphological plasticity as evidenced by the rapid resumption of the stratification process after such treatment is discontinued. Thus, short-term APB treatment of the developing retina delays, rather than permanently arrests, the formation of stratified RGC dendrites. Collectively, these findings demonstrate that glutamate-mediated afferent activity regulates a highly specific aspect of RGC dendritic restructuring during a relatively protracted developmental period.

\section{Materials and Methods}

Fetal and postnatal cats of known gestational ages were obtained from an in-house colony. Birth usually occurs on embryonic day 65 (E65), with the day after mating designated as E1. All procedures were in compliance with NIH standards and approved by the Animal Care and Use Committee of the University of California.

Surgical procedures. To obtain fetal retinas, timed pregnant cats were pretreated with atropine $(0.2 \mathrm{cc} / \mathrm{kg})$, and anesthetized with $4 \%$ halothane in oxygen. After intubation, a surgical plane of anesthesia was maintained with $1.0-2.0 \%$ Halothane and a mixture of $50 \%$ nitrous oxide, $50 \%$ oxygen. The uterine horns were exposed by a midline abdominal incision, a small opening was made over the nonplacental portion of the uterus through which the embryonic animals were removed for perfusion. Postnatal animals were obtained from timed-pregnant cats that came to parturition.

Intraocular injection. In experiments involving injections of APB, postnatal animals were premedicated with atropine and anesthetized with halothane. Intravitreal injections of $10 \mu \mathrm{l}$ containing $0.092 \mathrm{mg}$ APB diluted in sterile water were made daily with a Hamilton syringe through a 28 gauge needle. This dosage has been shown to be effective in suppressing ON-pathway responses under light-adapted conditions in the adult cat for as long as $24 \mathrm{hr}$ (Horton and Sherk, 1984). In all cases the injections were made in the temporal portion of the sclera at the level of the ora serrata. The animals received either $4,5,6,10$, or 11 injections of APB into one eye. The other eye was injected with an equal volume of sterile saline (vehicle control) or left untreated.

Tissue fixation and preparation. Six of the animals were perfused within $48 \mathrm{hr}$ of the last APB injection, while eight others were allowed to survive for $5,7,80$, or $140 \mathrm{~d}$. Following a lethal intraperitoneal injection of barbiturate, the animals were exsanguinated transcardially with $0.9 \%$ saline, followed by a $4 \%$ paraformaldehyde fixative. The eyes were removed intact from the cranium and placed for at least one week in fixative. Following postfixation, an incision was made in the optic nerve stump and crystals of the lipophilic tracer DiI $\left(1,1^{\prime}\right.$-dioctadecyl-3,3,3',3'-tetramethylindocarbocyanine perchlorate) were implanted within the nerve cut. After 7-10 weeks whole retinas were removed from the eye and mounted onto gelatinized slides. To obtain transverse sections, circular punches $3.5 \mathrm{~mm}$ in diameter were taken from the central (within $5 \mathrm{~mm}$ of area centralis) or peripheral retina. This tissue was embedded in $2 \%$ agar, sectioned in the transverse plane on a vibrotome at a $40 \mu \mathrm{m}$ thickness, mounted onto gelatinized slides, and coverslipped with Krystallon. Other DiI labeled whole-mounted retinas were photoconverted to stabilize the label. In preparation for photoconversion, the retinas were preincubated in a $1.5 \mathrm{mg} / \mathrm{ml}$ solution of 3,3' diaminobenzidine tetrahydrochloride (DAB) in $0.1 \mathrm{M}$ Tris- $\mathrm{HCl}$ buffer (TB), $\mathrm{pH} 8.2$, for up to $3 \mathrm{hr}$ at room temperature. After rinsing in TB, DiI labeled cells were photo-oxidized into a dark reaction product on a Nikon Optiphot microscope using a Nikon BA590 green fluorescent filter and a $10 \times$ objective. After photoconversion the retinas were briefly washed in TB, air dried overnight, and then coverslipped with DePex.

Data analysis. Transverse sections of DiI labeled RGCs were examined through a Nikon Optiphot microscope using a $50 \times$ fluorescent objective. Only cells whose dendritic processes appeared completely
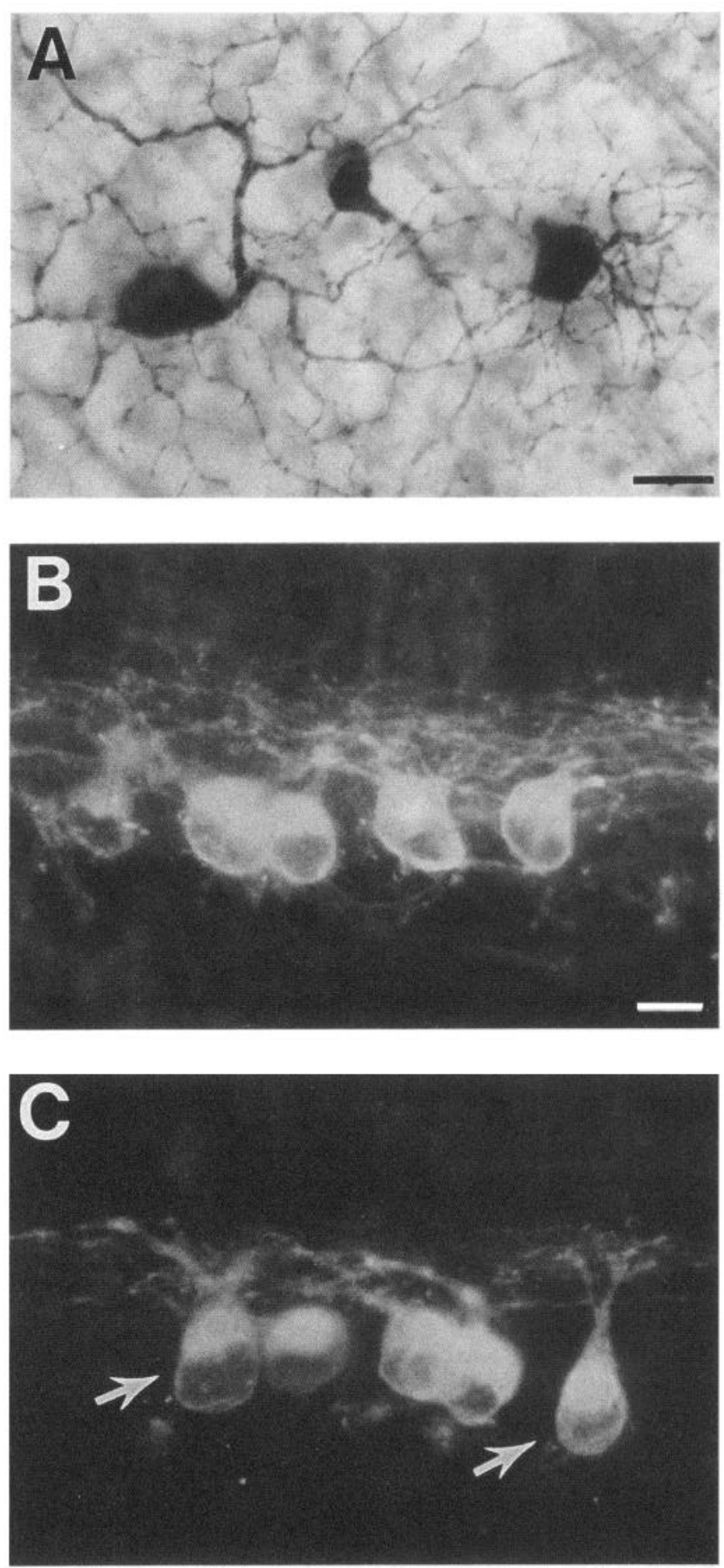

Figure 1. A, Photomicrograph of DiI labeled, photoconverted RGCs in an E56 wholemounted cat retina. An alpha (extreme left), beta (extreme right) and gamma (middle) cell can been identified. $B$, Photomicrograph of DiI labeled beta RGCs in transverse section of the central region of a normal E56 retina illustrating two pairs of beta cells with multistratified dendrites. $C$, Photomicrograph of five beta cells in a neighboring region of the central retina. The two cells denoted by $a r$ rows are characterized by unistratified dendrites, both terminating in the OFF sublamina of the IPL. Scale bars: $A, 20 \mu \mathrm{m} ; B$ and $C, 10 \mu \mathrm{m}$. 


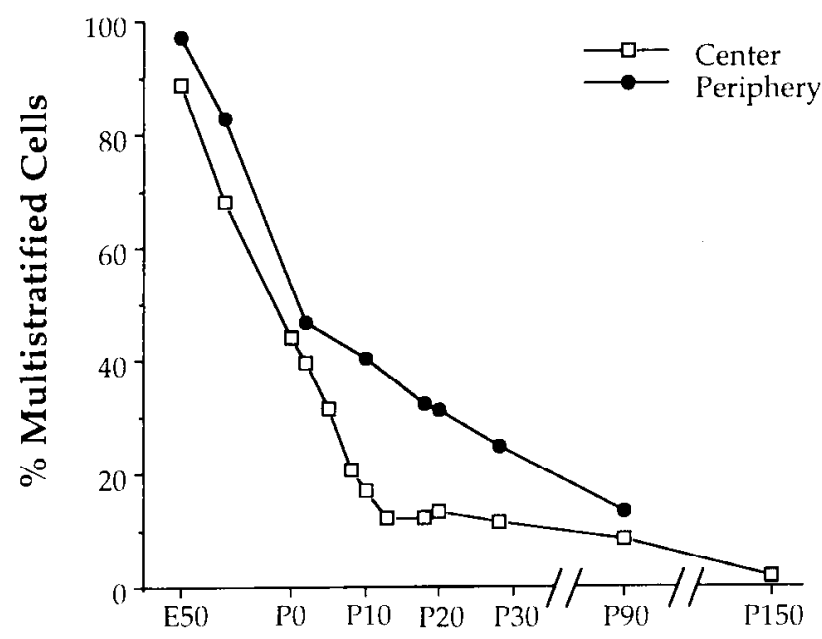

Age

Figure 2. Line graph depicting the time course of beta RGC dendritic stratification within central and peripheral regions of the retina from E50 to P150. At P150, only the central retina was analyzed duc to incomplete dendritic filling in the periphery. An average of 100 cells from at least two retinas were analyzed for each data point (range: 40 200 cells)

labeled with branching primary dendrites were included in the study. Branching was defined as a directional turn of more than $30^{\circ}$ of a primary dendrite within the IPL. Cells were identified as unistratified if the dendritic processes were confined entirely within the ON or OFF sublamina of the IPL, and multistratified if the dendrites extended into both sublaminae. The ON sublamina of the IPL was considered to be the half of this layer proximal to the RGC soma, whereas the OFF sublamina was considered to be the half distal to the soma.

In assessing the effects of APB treatment, the sections obtained from six retinas were examined initially without the observer's knowledge as to whether treated or control material was being analyzed. As described in the results, the APB-treated retinas (obtained from animals sacrificed within $48 \mathrm{hr}$ after the last APB injection) did not possess the two tiers of clearly defined dendritic strata evident in control material. Consequently, a true blind analysis could not be implemented. In several cases, the same material was assessed independently by two observers and this yielded nearly identical estimates of the incidence of multistratified cells.

Following photoconversion, retinal wholemounts were visualized through a Zeiss microscope, using a $40 \times$ oil immersion objective equipped with a camera lucida. Areal measurements of somata and dendritic fields were obtained from drawings of RGCs made on a digitizing tablet linked to a Macintosh computer and quantified with a graphics software package.

$P K C$ immunocytochemistry. For immunocytochemical identification of rod bipolar cells, APB-treated and control retinas were processed using a primary mouse monoclonal antibody to protein kinase $C$ (PKC) (Amersham). These retinas were perfusion-fixed as described above, cryoprotected in a $30 \%$ sucrose-phosphate buffer solution and then transverse-sectioned with a cryostat at a thickness of $10 \mu \mathrm{m}$. The sections were incubated overnight at $4^{\circ} \mathrm{C}$ in the primary antibody diluted 1:200 in PB containing 1\% BSA and 1\% Triton-X-100. Incubation in secondary IgG was for $90 \mathrm{~min}$, and incubation in $\mathrm{ABC}$ was for $60 \mathrm{~min}$, all at room temperature. The concentration of Triton X-100 in the incubation solutions was $0.5 \%$. After incubation in the $A B C$ solution, sections were rinsed in $\mathrm{PB}$, incubated in a DAB solution $(50-100 \mathrm{mg} /$ $100 \mathrm{ml} \mathrm{PB}$ ), and reacted by adding $3 \% \mathrm{H}_{2} \mathrm{O}_{2}$. The DAB reaction was terminated by rinsing in $\mathrm{PB}$. Some immunoreacted sections were counterstained in toluidine blue or cresyl violet. All sections were then dehydrated in a graded series of ethanols, cleared in xylene, and then coverslipped in DePex.

\section{Results}

The results are presented in three parts. First, we describe the time course of beta cell dendritic stratification in the central and

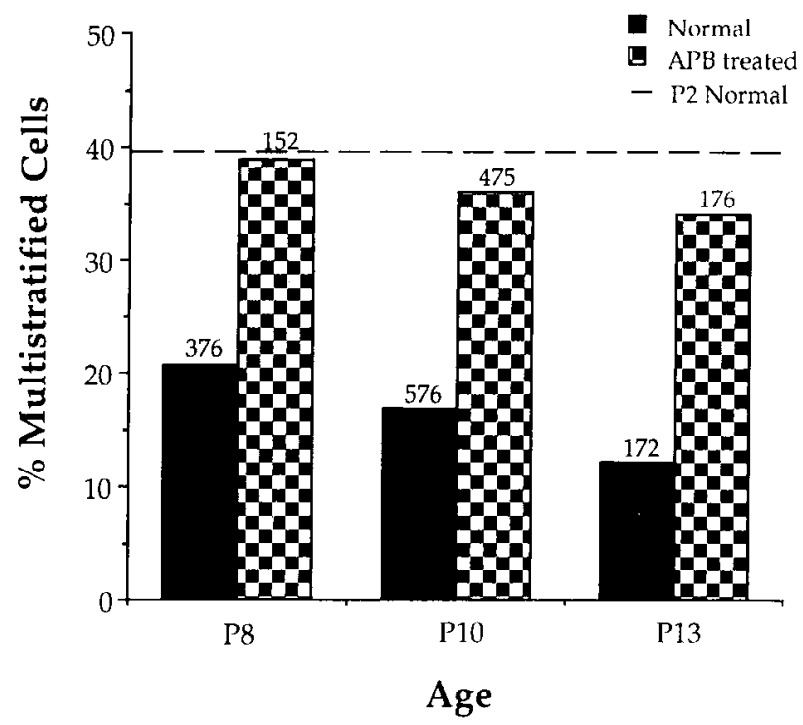

Figure 3. A comparison of the incidence of multistratified RGCs in normal and APB-treated retinas at three postnatal ages. The dotted line denotes the incidence of multistratified cells at $P 2$, the day when $A P B$ treatment was initiated. Two normal and two APB-treated retinas were analyzed at each age. The values listed above the bars indicate the number of RGCs analyzed. A statistical analysis of the difference in the incidence of multistratified cells in normal and APB-treated retinas showed that at all ages there were significantly more multistratified cells in APB-treated than in normal retinas (see Results for details).

peripheral regions of the retina. Second, we demonstrate that short-term APB intraocular injections during neonatal development arrests this stratification process, and that other salient aspects of retinal organization are not perturbed by such treatment. Third, we show that following termination of short-term APB treatment the stratification process resumes rapidly, resulting in a normal incidence of RGCs with unistratified dendrites.

\section{Time course of $R G C$ dendritic stratification}

Distinct classes of RGCs could be recognized in retinal wholemounts by E50, some 2 weeks before birth. This is in accord with the results of Ranna et al. (1988) who examined the nnorphological development of fetal cat RGCs labeled by intracellular injection of Lucifer yellow. As may be seen in Figure 1, beta cells could be readily distinguished from other RGC classes by their relatively short, bushy dendrites branching in close proximity to the cell body. Unequivocal identification of these cells could also be made in transverse sections which allowed for a more precise assessment of dendritic stratification in the developing IPL. The dendrites of labeled beta cells were identified as branching within one (unistratified) or more than one (multistratified) sublaminae of the IPL. Examples of beta cells with multistratified dendritic processes in transverse section are illustrated in an E56 retina in Figure $1 B$; the less frequently encountered unistratified beta cells at this age may be seen in Figure $1 C$.

The stratification of RGC dendrites is just beginning in both the central and the peripheral regions of the retina at E50 (Fig. 2 ). At this age we estimate that $89 \%$ of the cells are multistratified in the central retina, while $97 \%$ are in this state in the periphery. During the next 2 weeks, dendritic stratification proceeds rapidly and concurrently in the central and peripheral regions, so that near birth about $45 \%$ of the cells in the central and peripheral retina are multistratified. During the first postnatal 

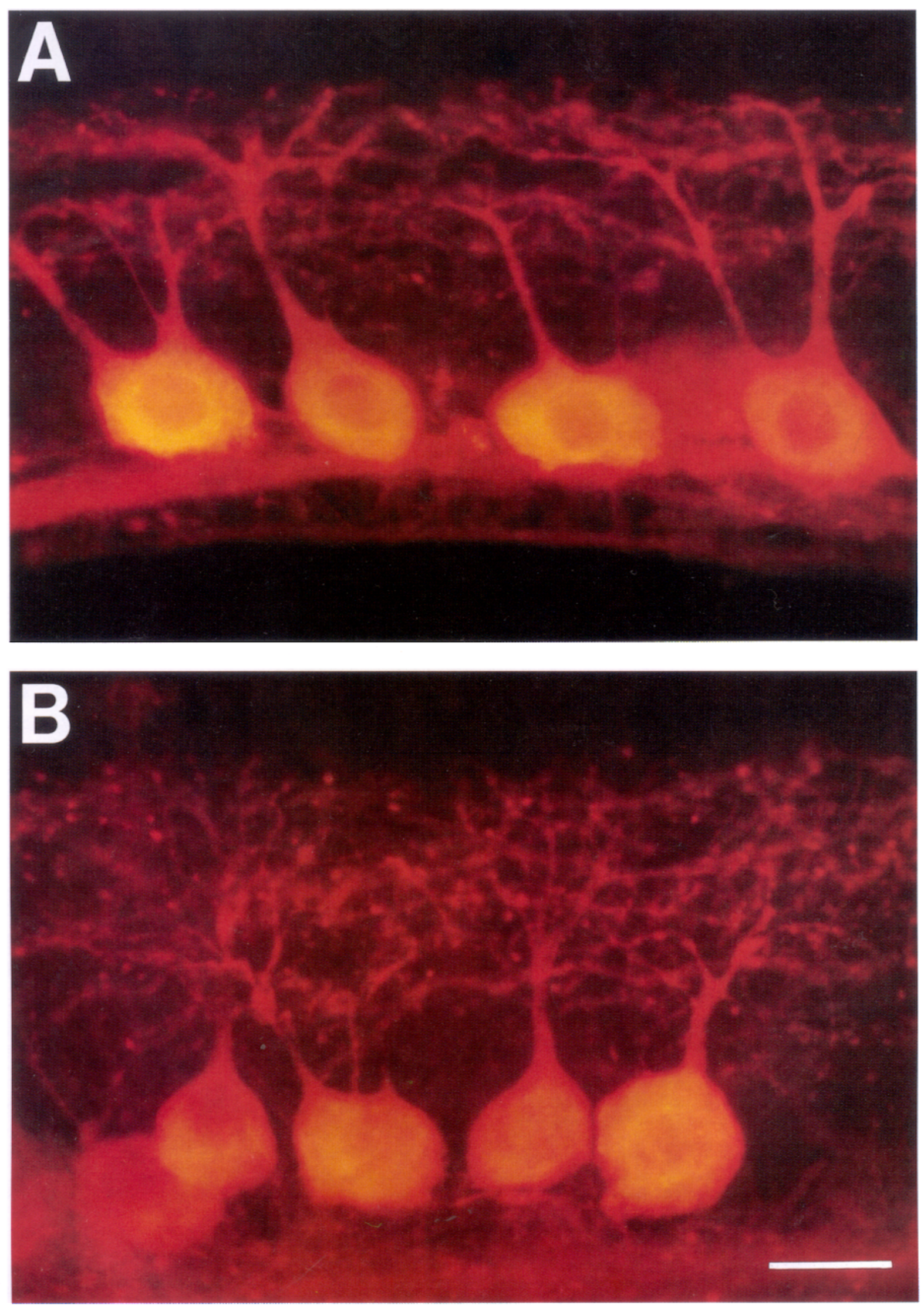

Figure 4. Photomicrographs showing DiI labeled RGCs in transverse section from equivalent central regions of a normal P13 (A) and an APBtreated P13 $(B)$ retina. In $A$, note the distinct appearance of two tiers of dendrites within the IPL and the stratification pattern of ON (proximal to the soma) and OFF (distal to the soma) RGC dendrites. In contrast, in $B$, the dendrites of individual cells remain multistratified, extending throughout the IPL in a pattern seen early in development in a normal retina. Scale bar, $20 \mu \mathrm{m}$. 
A

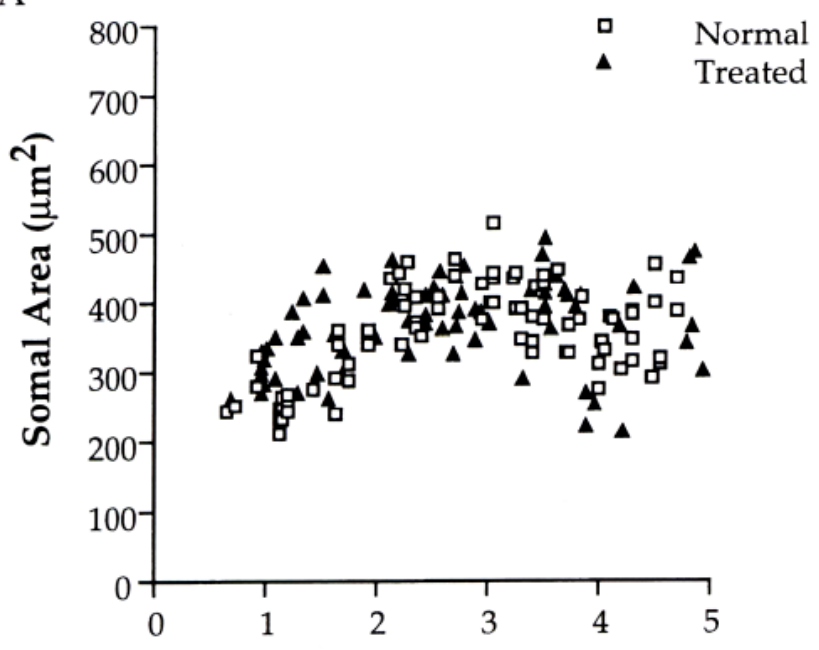

B

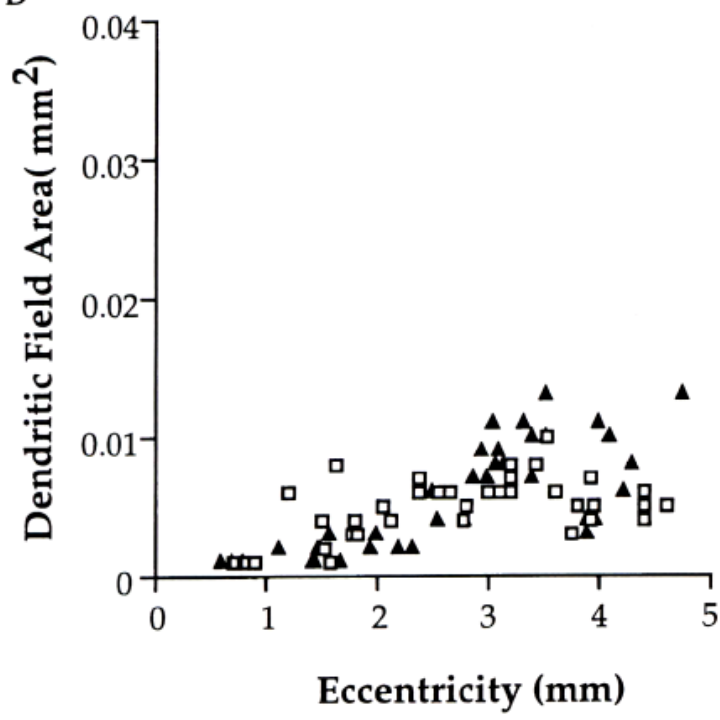

Figure 5. Scatter plots depicting the somal area $(A)$ and dendritic field area $(B)$ of beta RGCs at different retinal eccentricities $0-5 \mathrm{~mm}$ from the area centralis in normal and APB-treated retinas at P8. In both cases, there is extensive overlap of areal measurements taken from normal (squares) and treated (triangles) material at all eccentricities. There were no statistically significant differences between normal and APBtreated material.

week, the incidence of multistratified cells continues to decrease at a rapid rate within the central retina, while the rate of change in the periphery slows considerably. Consequently, a clear central-to-peripheral maturational gradient for this aspect of RGC restructuring first becomes apparent only several days after birth. This regional difference in the number of multistratified cells decreases with development, becoming negligible by $\mathrm{P} 90$, when $9 \%$ of the central cells and $13 \%$ in the periphery are still multistratified. By 5 months after birth virtually all beta cells have attained their mature unistratified state. There were no appreciable differences in the emergence of ON and OFF RGCs (data not illustrated).

\section{Effect of APB treatment}

To assess the possibility that glutamate-mediated afferent activity regulates the stratification process in developing RGCs, the
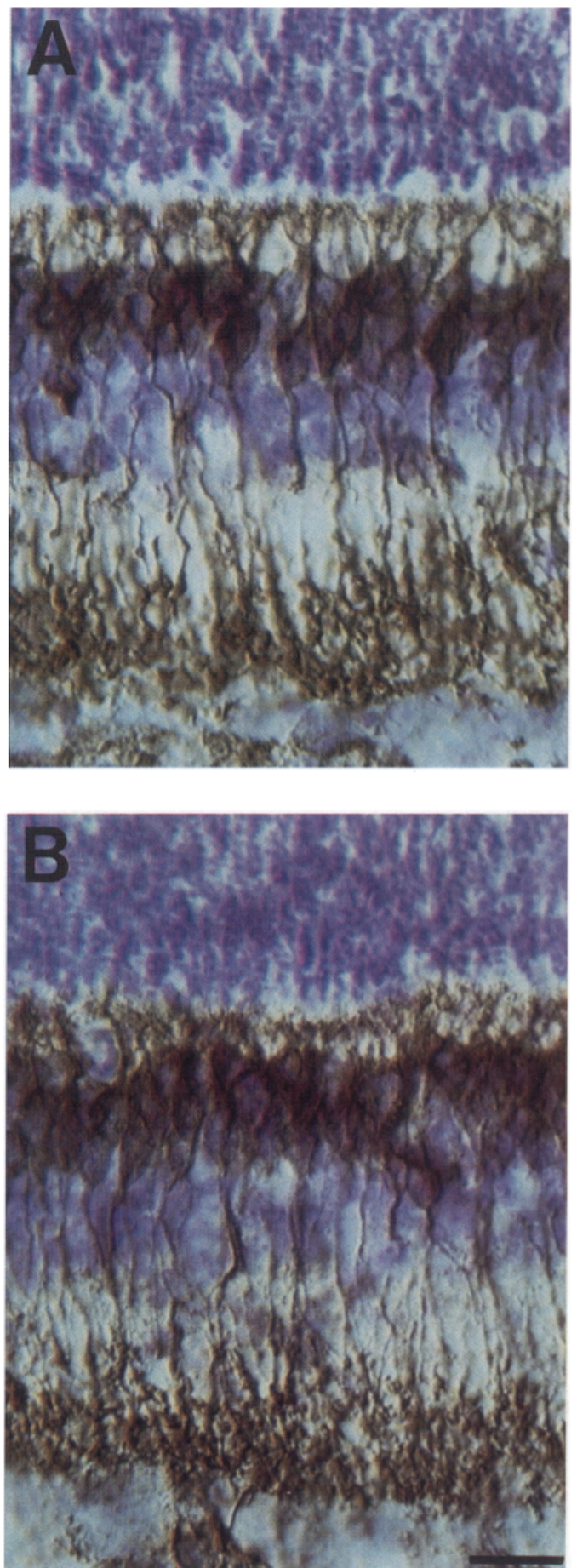

Figure 6. Photomicrographs showing normal $(A)$ and APB-treated $(B)$ retinas at P20 which were immunoreacted for protein kinase $C$ (PKC) and stained with cresyl violet. PKC immunoreactivity identified rod bipolar cells which, at maturity are known to be APB-sensitive. Note that the cytoarchitectural features of the INL and the level of PKC immunoreactivity do not differ in the normal and the APB-treated retinas. Scale bar, $20 \mu \mathrm{m}$.

glutamate analog APB was injected daily into one eye beginning at $\mathrm{P} 2$ for 5,7 , or $10 \mathrm{~d}$. These animals were sacrificed within 48 $\mathrm{hr}$ after the last injection. Two control and two APB-treated retinas were analyzed at each age. In all cases we confined the assessment of the incidence of multistratified cells to the central region of the retina, within $5 \mathrm{~mm}$ of the area centralis. Since 
there were no significant differences between normal control and vehicle control retinas, these data have been combined into a single comparison group denoted as "normal" in subsequent figures.

The consequences of APB treatment on the incidence of multistratified cells is illustrated in Figure 3. The dotted line denotes the incidence of cells $(39 \%)$ which are multistratified at P2, the day when APB treatment was initiated. As indicated by the dark bars the incidence of multistratified cells normally drops rapidly over the next $6 \mathrm{~d}$, so that by P8, about $21 \%$ of the cell are multistratified (see also Fig. 2). A more gradual reduction occurs during the next $5 \mathrm{~d}(17 \%$ at P10 and $12 \%$ at P13). In marked contrast, in the APB-treated retinas the proportion of multistratified cells remained at substantially higher levels at all ages examined. At P8 there were nearly twice as many multistratified cells in APB-treated retinas compared to normal (APB treated, $39 \%$; normal, $21 \%$ ). At P10, the APB-treated retinas contained $36 \%$ multistratified cells compared with $17 \%$ normal, and by P13, there were almost three times the number of multistratified cells (APB-treated, 34\%; normal, 12\%). In all three comparison groups these differences are statistically significant ( $G$ test for goodness of fit; all $G$ values significant at the $P<0.001$ level).

At all ages the incidence of multistratified cells in APB-treated retinas was not appreciably different than at P2, the age at which the injections were initiated. This indicates that during the early postnatal period APB treatment effectively arrests the normal stratification of beta RGC dendrites.

The photomicrographs in Figure 4 provide examples of DiIlabeled beta cells in transverse sections of a normal retina at P13 and an APB-treated retina of the same age. In the normal case (Fig. 4A), note the distinct appearance of two tiers of dendrites within the IPL and the stratification pattern of ON (proximal to the cell body) and OFF (distal to the cell body) RGCs. In striking contrast is the multistratified appearance of RGC dendrites within the IPL of the treated retina (Fig. 4B).

With the exception of the abnormally high incidence of multistratified cells, in both whole-mounts and transverse sections, the APB treated retinas appeared indistinguishable from normal material. To quantitatively assess the validity of this impression, we compared densities as well as somal and dendritic field sizes in the central regions of treated and control retinas. To compare cell densities, every Dil-labeled cell was counted, in two photoconverted whole-mounts, between an eccentricity of 1 and 2 $\mathrm{mm}$ from the area centralis. Cell densities were virtually the same in both cases (treated, 265 cells $/ \mathrm{mm}^{2}$; normal, 259 cells/ $\mathrm{mm}^{2}$ ) indicating that the APB treatment regimen was not toxic to developing RGCs. Thus, the increased incidence of multistratified cells observed in the treated retinas could not be due to a selective loss of stratified RGCs.

Somal and dendritic fields of beta cells were compared in normal and APB-treated P8 retinal wholemounts in which equivalent retinal regions contained exceptionally well-labeled cells. As may be seen in the scatter diagrams depicted in Figure 5, the distributions of normal and APB-treated somal (Fig. 5A) and dendritic field areas (Fig. $5 B$ ) overlapped extensively across the analyzed retinal regions. There were no significant differences in mean somal areas (normal, $356 \mu \mathrm{m}^{2}$; treated, $361 \mu \mathrm{m}^{2} ; t$ test: $1.98 ; p>0.05$ ) or mean dendritic field area (normal, 0.0047 $\mathrm{mm}^{2}$. treated: $0.0054 \mathrm{~mm}^{2} ; t$ test: $2.0 ; p>0.05$ ) between the normal and treated retinas. These measures of beta cell soma and dendritic field sizes are comparable to those reported by Dann et al. (1988) who studied Lucifer yellow filled RGCs in postnatal cats. Between $\mathrm{P} 2$ and $\mathrm{P} 8$ there is negligible normal growth of beta cell somal and dendritic field areas (Dann et al., 1988). Thus, our observations indicate that short-term APB treatment does not cause an atrophy of either beta cell somas or dendrites.

APB treatment also did not affect the cytoarchitectural features of the inner nuclear layer. In Nissl-stained transverse sections, the thickness of this layer and the density of cells appeared indistinguishable in APB-treated retinas in comparison to normal. The major class of APB-sensitive neurons within the INL, the rod bipolar cells, can be identified by protein kinase $\mathrm{C}$ (PKC) immunohistochemistry (Greferath et al., 1990). For this reason we compared the PKC staining pattern in APB-treated and normal retinas at P20. (At earlier ages, PKC expression is not normally prevalent in cat rod bipolar cells.) Photomicrographs of representative sections are illustrated in Figure 6, and as may be seen there is no difference in the density of PKC-immunoreactive rod bipolar cells or the quality of immunoreactivity between normal (Fig. 6A) and APB-treated (Fig. 6B) retinas. Thus, the cytoarchitectonic features of the INL, including the PKC-immunoreactivity of rod bipolar cells, were unaffected by APB intervention. This implies that the arrest of RGC dendritic stratification observed following APB treatment is not the result of gross morphological changes in the INL. Given the normal organization of the INL, it seemed reasonable to consider the possibility that the effects of short-term APB treatment could be reversed by allowing longer periods of survival.

\section{Recovery from $A P B$ treatment}

To assess the degree to which the effects of APB treatment are reversible, we examined the incidence of multistratified cells in APB-treated retinas following four different survival periods: 5 , 7, 80, and $140 \mathrm{~d}$. These injections were initiated at PO.

As may be seen in Figure 7, after recovery periods of 5 and $7 \mathrm{~d}$ the incidence of multistratified cells, although greater than in the normal retina of the same age, were significantly lower than in retinas of animals sacrificed within $48 \mathrm{hr}$ of the last APB injection. With longer recovery periods of 80 and $140 \mathrm{~d}$, the incidence of multistratified cells was found to be nearly equal in normal and APB-treated retinas. At P90, after APB treatment from $\mathrm{P} 0$ to $\mathrm{P} 10$ (11 injections) and $80 \mathrm{~d}$ of recovery, $11 \%$ of the beta RGCs were multistratified compared to $9 \%$ in the normal retinas. Following the same APB treatment regimen and 140 days of recovery, $6 \%$ of the cells were multistratified at P150 compared with $2 \%$ in normal retina. Thus, with long-term recovery, the incidence of multistratified cells decreased more than $30 \%$ after $\mathrm{P} 13$. In the normal retina there is only a $10 \%$ decrease in the incidence of such neurons after P13. These long-term recovery data demonstrate that postnatal RGCs retain a remarkable degree of morphological plasticity even after the normal stratification period is largely completed.

\section{Discussion}

In this study we used the DiI retrograde labeling technique to examine a key regressive event in the developing retina, the gradual restriction of RGC dendrites into ON or OFF sublaminae of the IPL. We have found that the stratification of beta cell dendrites begins about 2 weeks before birth and is fully completed by 5 months after birth. We also show that APB treatment, which perturbs the stratification of beta cell dendrites, does not affect the survival or the size of beta cell somas and dendrites. Furthermore, APB treatment does not appear to compromise the 


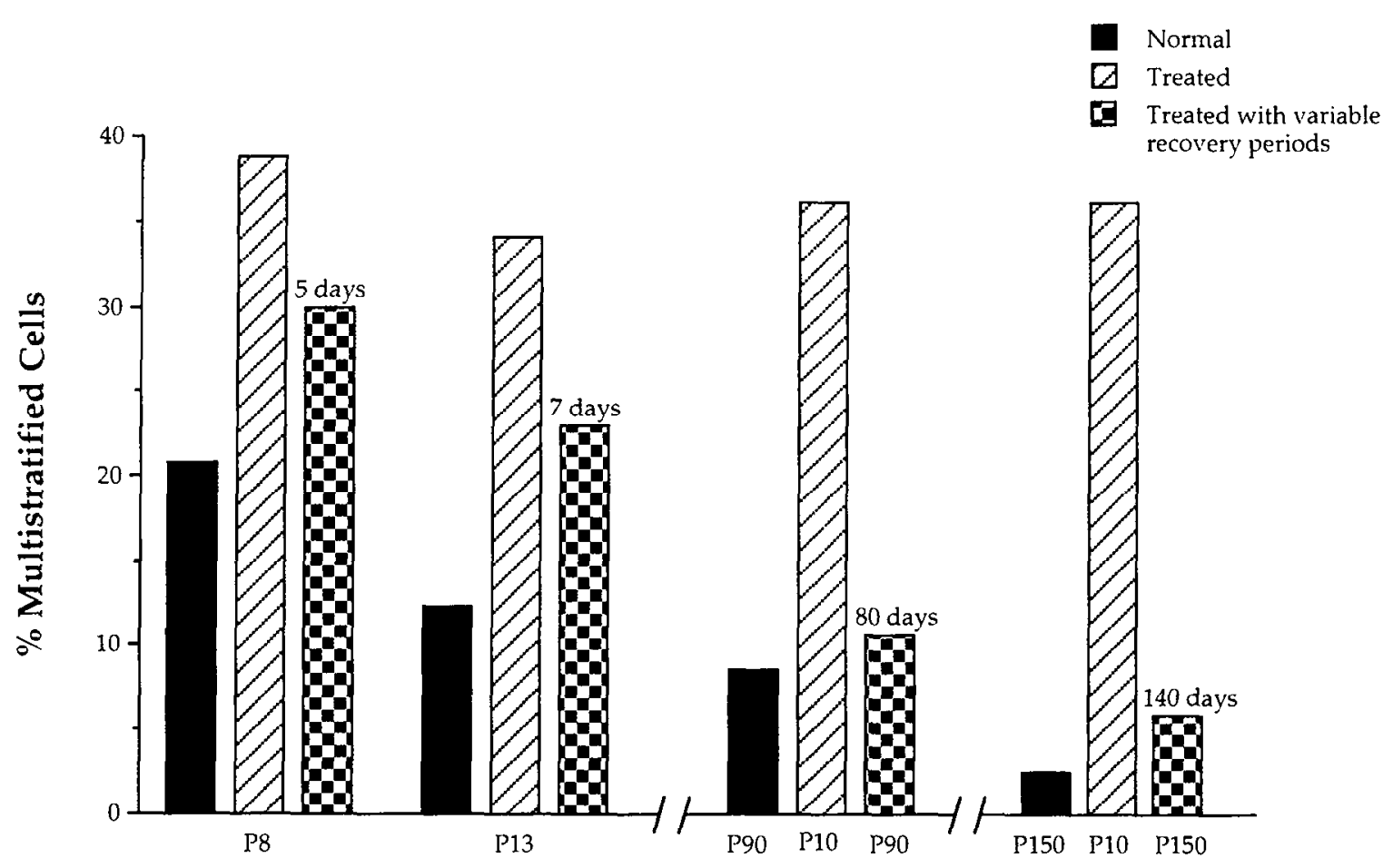

Age at Sacrifice

Figure 7. The effects of variable recovery periods after APB treatment on RGC dendritic stratification. The numbers on top of the recovery columns indicate the survival period following APB treatment. In the $5 \mathrm{~d}$ recovery group all animals were sacrificed at P8, while those in the $7 \mathrm{~d}$ recovery group were all sacrificed at P13. Normal and treated data were obtained from two retinas at each age. Recovery data are based on one retina at $5 \mathrm{~d}$ recovery, one at $7 \mathrm{~d}$, four at $80 \mathrm{~d}$, and two at $140 \mathrm{~d}$. In all cases, a minimum of 100 RGCs were analyzed for each data point.

organization of the INL, containing the APB-sensitive bipolar cells. The treated RGCs retained a remarkable degree of morphological plasticity as evidenced by the rapid resumption of the stratification process after APB treatment was discontinued, leading eventually to a normal incidence of stratified RGCs. Thus, short-term APB treatment of the neonatal cat retina causes a delay, rather than a permanent arrest, in the formation of stratified RGCs. Collectively, the findings imply that glutamate-mediated activity regulates a highly specific aspect of RGC development, and that such regulation occurs over a relatively protracted developmental period.

\section{Normal development of $R G C$ dendritic stratification}

The results demonstrate that beta cell dendritic stratification is already underway in both the central and peripheral retina at E50, more than 2 weeks before birth. Because morphological classes of RGCs are not differentiated until this period (Ramoa et al., 1988; and our observations), it was not possible to assess younger material. Given the low incidence of stratified cells at E50 (11\% in central retina and about $3 \%$ in periphery) it is seems likely that dendritic stratification begins no more than a few days earlier. Before birth there is only a small difference between central and peripheral retinal regions in the incidence of stratified beta cells. The significant central-to-peripheral gradient in stratified cells, which first becomes evident in the neonatal retina, is due to the more rapid rate of change in the central region. What causes the accelerated stratification process is unknown, but this could be due to a higher level of synaptogenesis in the neonatal central retina in comparison to the periphery (Maslim and Stone, 1986).

\section{$A P B$ treatment and the development of dendritic stratification}

Intraocular injection of APB during a relatively brief postnatal period disrupted the formation of structurally segregated $O N$ and OFF RGCs. In the mature cat retina, only ON cone bipolar cells and rod bipolar cells are APB sensitive (Müller et al., 1988). The application of this glutamate analog hyperpolarizes these retinal interneurons thereby preventing their release of glutamate. Thus, our results imply that stratification of $\mathrm{ON}$ and $\mathrm{OFF}$ RGCs in the developing cat retina depends on glutamate-mediated afferent activity.

There was no indication in our data that APB treatment selectively affected the ON pathway (see also Table 1 in Bodnarenko and Chalupa, 1993), even though OFF bipolar cells are not APB sensitive. Most likely this reflects the fact that APB-sensitive rod bipolar cells provide indirect inputs to both $O N$ and OFF RGCs. Rod bipolar cells terminate on AII amacrine cells and these interneurons connect with ON cone bipolar cells via a gap junction and a conventional synapse with OFF RGCs (Kolb and Famiglietti, 1974; Famiglietti and Kolb, 1975; McGuire et al., 1984, 1986). Prolonged hyperpolarization of the rod bipolar cells by APB attenuates afferent activity to all RGCs so that under scotopic conditions application of $\triangle \mathrm{PB}$ completely eliminates visual responses of all ON and OFF RGCs in the cat retina (Wässle et al., 1991). In the rod-dominated cat retina, the relatively small number of presumably unaffected OFF cone bi- 
Figure 8. A schematic diagram illustrating an asymmetric afferent innervation pattern as a possible mechanism for RGC dendritic stratification.
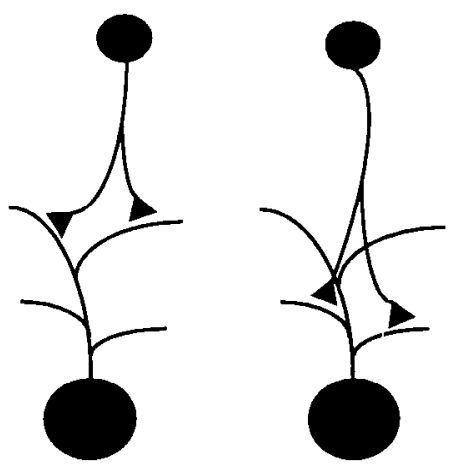

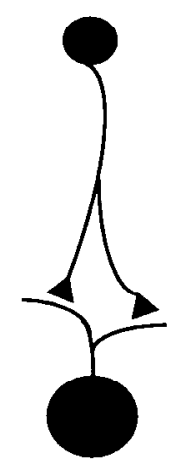

polar cells apparently were incapable of inducing retraction of OFF RGCs in the APB-treated retinas.

A key assumption of this line of reasoning is that developing bipolar cells respond to APB in a similar manner as at maturity. As yet, however, nothing is known about the sensitivity of developing bipolar cells to APB; nor can we rule out the possibility that, unlike in the mature retina, other cell types might be APBsensitive in the neonatal retina. Whole-cell patch clamp studies dealing with responsivity to APB in developing cat retinal cells (now in progress in our laboratory) would be useful in this regard.

The arrest of dendritic stratification obtained by APB treatment appears to be a specific phenomenon from two different perspectives. First, our observations indicate that APB treatment, which arrests dendritic stratification, did not cause any appreciable change in the somal size or the tangential extent of dendrites, nor did it result in an abnormal loss of RGCs. Furthermore, the gross morphological features of the INL also appeared normal, including the expression of PKC immunoreactivity in rod bipolar cells, the numerically largest population of APBsensitive neurons. Conceivably, a more detailed examination (e.g., at the ultrastructural level) could reveal additional consequences of APB treatment on retinal circuitry, but this remains to be established. Second, it is also the case that other manipulations of activity levels in the developing retina have been reported to be ineffective in disrupting the dendritic stratification of developing RGCs. In particular, intraocular TTX injections, which disrupt refinements of retinogeniculate terminal arbors, do not perturb the normal restriction of $\mathrm{RGC}$ dendritic processes (Dubin et al., 1986; Wong et al., 1991). Early monocular deprivation also does not perturb the normal formation of stratified RGC dendrites (Leventhal and Hirsch, 1983; Lau et al., 1990). Collectively, these observations indicate that it is not $\mathrm{RGC}$ activity per se which regulates the stratification process. This means that the phenomenon we arc dealing with cannot bc readily explained by a Hebbian-type mechanism which relies on preand postsynaptic temporally correlated spike discharges.

The effect we observed with APB treatment provides the first demonstration that retinal afferents regulate the normal segregation of RGC dendrites into $\mathrm{ON}$ and $\mathrm{OFF}$ sublaminae of the IPL. The mechanisms underlying this phenomenon are unknown. One possibility is that there is an asymmetrical distribution of synaptic inputs favoring either distal or proximal dendritic processes of initially multistratified RGCs. If this were the case, then an activity-mediated afferent input could instruct these neurons which dendritic process to retract and which to maintain. Either the distal or proximal process activated by synaptic inputs would be maintained, while the dendritic process lacking such contacts, or with insufficient contacts, would be withdrawn. Such an "instructional" mechanism is depicted schematically in Figure 8. This model provides a parsimonious explanation of how blockade of afferent activity could perturb the stratification process, and it also accounts for the ineffectiveness of TTX treatment and monocular deprivation. It should be emphasized that RGC dendritic stratification begins before birth, and for this reason visual input cannot play a significant role in this process. Rather, our results imply that the relevant afferent signal is the release of glutamate by bipolar cells due to intrinsic retinal activity. ON-cone bipolar cells could regulate the stratification process via direct synaptic contacts with RGCs. On the other hand, the influence of rod bipolar cells could be mediated indirectly via AII amacrines. Additionally, amacrine cell inputs could contribute to the regulation of the stratification process without the involvement of bipolar cells. The latter mode of action may be especially prevalent early in the stratification process since amacrine cells have been reported to form synaptic contacts with RGCs before such contacts are formed by bipolar cells (Maslim and Stone, 1986).

The model illustrated in Figure 8 assumes that the initial innervation of RGCs by retinal afferents is normally quite specific, in that, the synaptic contacts of an individual multistratified ganglion cell would be largely confined to one or the other strata of the developing IPL. As yet, however, nothing is known about the ingrowth of bipolar cell terminals, nor do we have any information of how APB treatment affects bipolar and amacrine cell terminal lamination within the IPL. These points remain to be addressed in future experiments.

An alternative explanation, not requiring asymmetric innervation, is that afferent activity acts to simply trigger an intrinsic program in developing RGCs leading to the retraction of dendrites from one or the other strata of the IPL. To account for the formation of ON and OFF RGCs, it would need to be the case that ganglion cells become differentiated into ON and OFF subtypes prior to the time of their retraction of multistratified dendritic processes.

The relative merit of the two alternative explanations we have proposed could be assessed by examining the distribution of synaptic contacts onto multistratified dendrites of developing RGCs. If the early synaptic contacts were asymmetrical onto distal and proximal dendritic processes this would lend support for the instructional model; whereas unbiased synaptic distributions onto multistratified processes would be in line with the intrinsic program hypothesis.

If our interpretation that glutamate-mediated activity is responsible for the stratification process is correct, it follows that blocking glutamate receptors on RGCs, during the same devel- 
opmental period, should also perturb dendritic stratification. In the developing hamster retina, intraocular injections of the NMDA receptor blocker APV has been reported to prevent the transient elimination of dendritic spines on RGC's (Lau et al., 1992), but in this study the state of stratified dendrites was not examined. Synaptic contacts are rarcly made on transicnt dendrites (Wong et al., 1992), so the relation of this observation to the issue of afferent inputs is obscure.

\section{Recovery from short-term $A P B$ treatment}

After APB treatment was discontinued there was a rapid resumption of RGC dendritic stratification, with full recovery occurring within 80 days. This demonstrates that short-term APB treatment only temporarily arrests the stratification of developing RGC dendrites. With the reinslatement of "normal" retinal activity developing RGCs showed a remarkable degree of morphological plasticity. This indicates that the chronological age of these cells is not the limiting factor in the stratification process. Because dendritic stratification of the entire population of RGCs is normally not completed until several months after birth, it would now be of considerable interest to assess the consequence of long-term APB treatment. Such an approach could reveal a developmental window constraining the recovery period, analogous to what has been demonstrated in the monocular deprivation literature. The consequences of short-term monocular deprivation can be entirely reversed by normal experience, whereas the effects of long-term deprivation are largely irreversible (Movshon and Van Sluyters, 1981). Similarly, long-term APB treatment, extending into the period when the stratification process has been normally largely completed, could well produce a permanent arrest in the multistratified state. Experiments exploring this possibility are now in progress.

\section{Relation to formation of $R G C$ mosaic patterns}

In the mature cat retina, ON and OFF RGCs of the alpha and beta classes are distributed in regular mosaic patterns, with the dendrites of each subclass uniformly tiling the retina (Wässle et al., 1981). Such an organization is thought to provide an efficient sampling of the visual world by functionally distinct RGCs encoding responses to increments and decrements of light. A key step in the establishment of such ON and OFF retinal pathways is the stratification of RGC dendrites, described for beta cells in the present study. As yet, we do not know when such RGC mosaics become established. There is reason to think, however, that even after the stratification process is largely completed, the distributions of $\mathrm{ON}$ and $\mathrm{OFF}$ RGCs in the postnatal retina are less regular than at maturity. This is because more than 100,000 RGCs are normally eliminated during postnatal development of the cat retina (Williams ct al., 1986), and these "excess" cells could obscure regular mosaic patterns. Thus, the formation of RGC mosaics probably involves two largely separate developmental events: the stratification of RGC dendrites, which we have shown to be mediated by glutamate afferent activity, and a selective regional loss of RGC cells, controlled by factors which remain to be elucidated.

\section{References}

Bodnarenko SR, Chalupa LM (1993) Stratification of ON and OFF ganglion cell dendrites depends on glutamate-mediated afferent activity in the developing retina. Nature 364:144-146.
Bolz J, Wässle H, Thier P (1984) Pharmacological modulation of on and off ganglion cells in the cat retina. Neuroscience 12:875-885.

Dann J, Buhl E, Peichl L (1988) Postnatal dendritic maturation of alpha and beta ganglion cells in cat retina. J Neurosci 8:1485-1499.

Dubin M, Stark L, Archer S (1986) A role for action-potential activity in the development of neuronal connections in the kitten retinogeniculate pathway. J Neurosci 6:1021-1036.

Famiglietti EV, Kolb H (1975) A bistratified amacrine cell and synaptic circuitry in the inner plexiform layer of the retina. Brain Res 84:293300 .

Famiglietti EV, Kolb H (1976) Structural basis for ON- and OFF-center responses in retinal ganglion cells. Science 194:193-195.

Greferath U, Grünert U, Wässle H (1990) Rod bipolar cells in the mammalian retina show protein kinase C-like immunoreactivity. J Comp Neurol 301:433-442.

Horton J, Sherk H (1984) Receptive field properties in the cat's lateral geniculate nucleus in the absence of on-center retinal input. $J$ Neurosci 4:374-380.

Kalil R, Dubin M, Scott G, Stark L (1986) Elimination of action potentials blocks the structural development of retinogeniculate synapses. Nature 323:156-158.

Kolb H, Famiglietti EV (1974) Rod and cone pathways in the inner plexiform layer of the cat retina. Science 186:47-49.

Lau K, So K, Tay D (1990) Effects of visual or light deprivation on the morphology and the elimination of the transient features during development of type I retinal ganglion cells in hamsters. J Comp Neurol 300:583-592.

Lau K, So K, Tay D (1992) APV prevents the elimination of transient dendritic spines on a population of retinal ganglion cells. Brain Res 595:171-174.

Leventhal A, Hirsch H (1983) Effects of visual deprivation upon the morphology of retinal ganglion cells projecting to the dorsal lateral geniculate nucleus of the cat. J Neurosci 3:332-344.

Maslim J, Stone J (1986) Synaptogenesis in the retina of the cat. Brain Res 373:35-48

Maslim J, Stone J (1988) Time course of stratification of the dendritic fields of ganglion cells in the retina of the cat. Dev Brain Res 44: 87-93.

McGuire B, Stevens J, Sterling P (1984) Microcircuitry of bipolar cells in cat retina. J Neurosci 4:2920-2938.

McGuire B, Stevens J, Sterling P (1986) Microcircuitry of beta ganglion cells in the cat retina. J Neurosci 6:907-918.

Movshon J, Van Sluyter RC (1981) Visual neural development. Annu Rev Psychol 32:477-522.

Müller F, Wässle H, Voigt T (1988) Pharmacological modulation of the rod pathway in the cat retina. J Neurophysiol 59:1657-1672.

Nelson R, Famiglietti EV, Kolb H (1978) Intracellular staining reveals different levels of stratification for on- and off-center ganglion cells in cat retina. J Neurophysiol 41:472-483.

Ramoa A, Campbell G, Shatz CJ (1988) Dendritic growth and remodeling of cat retinal ganglion cells during fetal and postnatal development. J Neurosci 8:4239-4261.

Shatz CJ, Stryker M (1988) Prenatal tetrodotoxin infusion blocks segregation of retinogeniculate afferents. Science 242:87-89.

Slaughter M, Miller R (1981) 2-Amino-4-phosphonobutyric acid: a new pharmacological tool for retina research. Science 211:182-185.

Sretavan D, Shatz CJ, Stryker M (1988) Modification of retinal ganglion cell axon morphology by prenatal infusion of tetrodotoxin. Nature 336:468-471.

Wässle H, Boycott B, Illing R (1981) Morphology and mosaic of onand off-beta cells in the cat retina and some functional considerations. Proc R Soc Lond [Biol] 212:177-195.

Wässle H, Yamashita M, Greferath U, Grünert U, Müller F (1991) The rod bipolar cell of the mammalian retina. Visual Neurosci 7:99-112.

Wässle H, Boycott B (1991) Functional architecture of the mammalian retina. Physiol Rev 71:447-480.

Williams R, Bastiani M, Lia B, Chalupa LM (1986) Growth cones, dying axons, and developmental fluctuations in the fiber population of the cat's optic nerve. J Comp Neurol 246:32-69.

Wong ROL, Herrmann K, Shatz CJ (1991) Remodeling of retinal ganglion cell dendrites in the absence of action potential activity. J Neurobiol 22:685-697.

Wong ROL, Yamawaki, RM, Shatz CJ (1992) Synaptic contacts and the transient dendritic spines of developing retinal ganglion cells. Eur J Neurosci 4:1387-1397. 